\title{
Plasmodium falciparum hydroxymethylbilane synthase does not house any cosynthase activity within the haem biosynthetic pathway
}

\author{
Alan F. Scott ${ }^{1, *}$, , Evelyne Deery ${ }^{1}$, Andrew D. Lawrence ${ }^{1}$ and Martin J. Warren ${ }^{1,2}$
}

\begin{abstract}
Uroporphyrinogen III, the universal progenitor of macrocyclic, modified tetrapyrroles, is produced from aminolaevulinic acid (ALA) by a conserved pathway involving three enzymes: porphobilinogen synthase (PBGS), hydroxymethylbilane synthase (HmbS) and uroporphyrinogen III synthase (UroS). The gene encoding uroporphyrinogen III synthase has not yet been identified in Plasmodium falciparum, but it has been suggested that this activity is housed inside a bifunctional hybroxymethylbilane synthase (HmbS). Additionally, an unknown protein encoded by PF3D7_1247600 has also been predicted to possess UroS activity. In this study it is demonstrated that neither of these proteins possess UroS activity and the real UroS remains to be identified. This was demonstrated by the failure of codon-optimized genes to complement a defined Escherichia coli hemD- mutant (SASZ31) deficient in UroS activity. Furthermore, HPLC analysis of the oxidized reaction product from recombinant, purified $P$. falciparum HmbS showed that only uroporphyrin I could be detected (corresponding to hydroxymethylbilane production). No uroporphyrin III was detected, showing that P. falciparum HmbS does not have UroS activity and can only catalyze the formation of hydroxymethylbilane from porphobilinogen.
\end{abstract}

\section{INTRODUCTION}

Haem, as an iron-containing porphyrin, is a modified tetrapyrrole that is derived from the starting material 5-aminolevulinic acid (5-ALA) [1]. The construction of the macrocyclic framework of haem is mediated in just three steps [1]. Firstly, two molecules of 5-ALA are condensed to give a pyrrole, porphobilinogen (PBG), in a reaction catalyzed by PBG synthase $[2,3]$. The next step involves the polymerization of four pyrrole units (termed A-D) into a linear bilane called hydroxymethylbilane (HMB) and is mediated by an enzyme called HMB synthase (HmbS) that deaminates and links together four molecules of PBG [4-7]. Finally, the bilane undergoes cyclization, but only after inversion of the terminal D ring, to give uroporphyrinogen III $[6,7]$. These three steps are found in all organisms that make modified tetrapyrroles [1]. These reactions are shown in Fig. 1.

A pathway for haem biosynthesis is found in Plasmodium falciparum, the protozoan parasite and causative agent of malaria [8]. As a haematophagous organism (blood-feeding parasite), it exists for part of its life cycle in a haem-rich environment and releases large quantities of haem as an insoluble crystalline material called haemozoin [9]. Nonetheless, it requires a functional haem synthesis pathway for survival in the liver and mosquito growth stages [10,11]. Recent studies have biochemically characterized the complete set of haem synthesis enzymes from $P$. falciparum with the notable exception of uroporphyrinogen III synthase (UroS, formerly HemD) [12-18]. This enzyme is sometimes called uroporphyrinogen III cosynthase, as it often co-purifies with hydroxymethylbilane synthase (HmbS, formerly porphobilinogen deaminase or HemC), and both of these enzymes are required to make uroporphyrinogen III from PBG [5, 19]. HmbS catalyzes the synthesis of an unstable linear tetrapyrrole, $\operatorname{HMB}[4,6,7]$. This rapidly cyclizes into uroporphyrinogen I unless the cosynthase is present to invert the terminal ring and cyclize HMB into uroporphyrinogen III $[6,7,20]$. This is

Received 16 February 2021; Accepted 19 August 2021; Published 18 October 2021

Author affiliations: 'School of Biosciences, University of Kent, Canterbury, Kent, CT2 7NJ, UK; ${ }^{2}$ Quadram Institute Bioscience, Norwich Research Park, Norwich, NR4 7UQ, UK.

*Correspondence: Alan F. Scott, scottA11@cardiff.ac.uk

Keywords: haem synthesis; hydroxymethylbilane; porphobilinogen deaminase; Plasmodium falciparum; uroporphyrinogen III.

Abbreviations: ALA, amino levulinic acid; HMB, hydroxymethylbilane; HmbS, hydroxymethylbilane synthase; LB, Lysogeny Broth; PBG,

porphobilinogen; UroS, uroporphyrinogen III synthase.

†Present address: School of Chemistry, Cardiff University, Cardiff, CF10 3AT, UK.

Six supplementary figures are available with the online version of this article.

001095 @ 2021 The Authors 


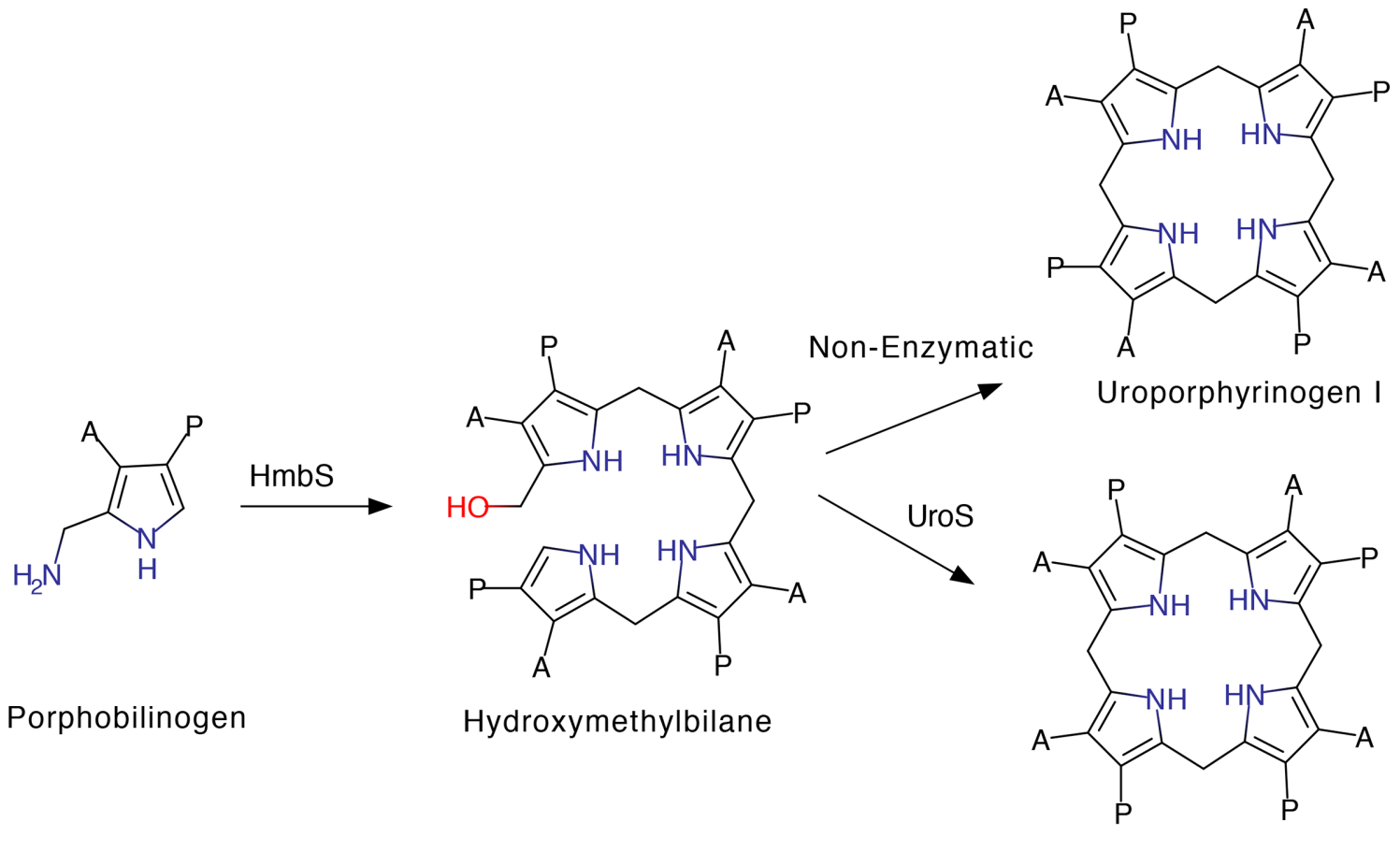

Uroporphyrinogen III

Fig. 1. Reactions of $\mathrm{HmbS}$ and UroS. HmbS polymerizes porphobilinogen into hydroxymethylbilane, which auto-cyclizes to uroporphyrinogen I. If UroS is present, then hydroxymethylbilane is cyclized into uroporphyrinogen III, a reaction that involves the inversion of ringD. A, acetic acid; $P$, propionic acid.

the only isomer that can proceed through the haem synthesis pathway. A candidate gene encoding UroS in P. falciparum (PF3D7_1247600) has been identified by bioinformatics, but there have been no biochemical studies to validate the finding [21]. Another report in the literature has suggested that the parasite does not need a separate cosynthase because UroS activity can be found within a bi-functional $\mathrm{HmbS}$ that houses both HMB synthase and uroporphyrinogen III cosynthase activities [18]. The evidence presented for this was HPLC identification of the (oxidized) reaction product as uroporphyrin III from both native and recombinant $\mathrm{HmbS}$ when incubated with PBG.

Although such dual activity has previously been reported for HmbS from Leptospira interrogans, this is a very different protein from $P$. falciparum $\mathrm{HmbS}$, being a fusion of $\mathrm{HmbS}$ and UroS enzymes [22]. Conversely, the P. falciparum HmbS is clearly not a fusion protein because it has similarity to other HmbS enzymes throughout its entire sequence length (with the exception of the N-terminal apicoplast localization sequence, but dual activity was claimed for a truncated HmbS without this signal sequence) [18]. There are some short inserts in the P. falciparum sequence, but it is unlikely that UroS activity is contained within these inserts because they are not very long - the longest is 31 amino acids. The sequence does not align to known UroS sequences. A multiple sequence alignment is shown in Figs S1 and S2 (available in the online version of this article). It is, therefore, hard to understand how this enzyme could house two very different activities. Consequently, this report investigates more closely the evidence for dual activity using genetic complementation studies and analytical chemistry. The possibility that UroS activity is encoded by PF3D7_1247600 is also investigated.

\section{EXPERIMENTAL PROCEDURES Gene cloning}

Synthetic, codon-adapted genes, based on PF3D7_1209600 (hemC) and PF3D7_1247600 (putative hemD), were purchased from GeneArt for optimal expression in Escherishia coli (Fig. S3) and subcloned into a $p E T-3 a$ vector (Novagen) using NdeI and SpeI restriction sites (the pET-3a had been modified to include a SpeI site $5^{\prime}$ of the BamHI site). Two further constructs were made containing a truncated version of the hem $C$ gene to remove a potential signal sequence from the protein product [18]. The truncated gene was obtained by PCR using the following primers: $5^{\prime}$ primer containing NdeI site and start codon: CACCATATGGGCATCAAAGAT GAAATTATTATCGG; 3' primer containing SpeI site and stop codon: ctcactagttatttattgttcagcagg.

The PCR product was ligated into $p E T-3 a$ and $p E T-14 b$ (Novagen) usingNdeI and SpeI restriction sites (both vectors had been previously modified to include a SpeI site $5^{\prime}$ of the BamHI site). 


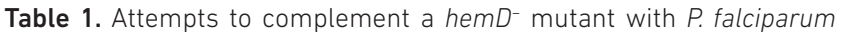
hemc

\begin{tabular}{|c|c|c|c|}
\hline Construct & $24 \mathrm{~h}$ growth & $48 \mathrm{~h}$ growth & $\begin{array}{l}\text { Restreaked } \\
24 \mathrm{~h} \text { growth }\end{array}$ \\
\hline pET-3a & + & ++ & - \\
\hline pET-14b & + & ++ & + \\
\hline $\begin{array}{l}\text { pET-3a hemC } \\
\text { (P. falciparum) }\end{array}$ & + & ++ & - \\
\hline $\begin{array}{l}\text { pET-3a hemC } C^{\text {truncated }} \\
\text { (P. falciparum) }\end{array}$ & + & ++ & - \\
\hline $\begin{array}{l}\text { pET-3a hemD } \\
\text { (P. falciparum) }\end{array}$ & + & ++ & + \\
\hline $\begin{array}{l}\text { pET-14b hemD } \\
\text { (E. coli) }\end{array}$ & ++++ & ++++ & ++++ \\
\hline $\begin{array}{l}\mathrm{pET}-14 \mathrm{~b} \text { hemD } \\
\text { (B. megaterium) }\end{array}$ & ++++ & ++++ & ++++ \\
\hline
\end{tabular}

hemD' $D^{-}$mutant SASZ31 was transformed with various constructs and incubated on LB agar plates with $0.2 \%$ glucose and appropriate antibiotics at $37^{\circ} \mathrm{C}$ for $48 \mathrm{~h}$. The size of any resultant colonies was recorded after 24 and $48 \mathrm{~h}$. To test for viability, the colonies were restreaked onto a fresh plate and grown for a further $24 \mathrm{~h}$ and examined for evidence of growth. The growth is indicated in the table by the number of plus signs from + (poor growth) to ++++ (normal growth). A indicates that no growth was observed.

The constructs were sequenced by GATC Biotech to check for the correct insert and reading frame and the absence of mutations.

\section{Complementation studies}

A defined hemD ${ }^{-}$mutant SASZ31 (CGSC\# 7153 Coli Genetic Stock Center, Yale University) [23] was transformed with the following plasmids: pET-3a empty vector; pET-14b empty vector; pET-3a hemD P. falciparum 3D7_1247600; pET-3a hemC P. falciparum hemC; pET-3a hem $C^{\text {truncated }}$ P. falciparum hemC Cruncated; pET-14b hemD B. megaterium hemD [24]; pET14b hemD E. coli hemD (a kind gift from Professor Peter Shoolingin-Jordan, Southampton).

The transformations were plated onto LB (lysogeny broth) agar plates with $100 \mu \mathrm{g} \mathrm{ml}^{-1}$ ampicillin and $2 \%$ glucose and incubated at $37^{\circ} \mathrm{C}$ for $24 \mathrm{~h}$. They were examined for growth and left for an additional $24 \mathrm{~h}$, after which the colonies were restreaked onto fresh plates. After incubation at $37^{\circ} \mathrm{C}$ for $24 \mathrm{~h}$ the plates were examined for growth.

\section{Protein overproduction and purification}

BL21 $1^{\text {STAR }}$ (DE3) pLysS (Invitrogen) was transformed with the appropriate construct and a 11 culture of the resulting strain was grown in $\mathrm{LB}$ at $37^{\circ} \mathrm{C}$ with shaking to an $\mathrm{OD}_{600}$ of 0.6 . Gene expression was induced for $20 \mathrm{~h}$ at $19^{\circ} \mathrm{C}$ by adding $0.4 \mathrm{mM}$ IPTG. Cells were harvested by centrifugation at 4000 r.p.m. for $15 \mathrm{~min}$ at $4^{\circ} \mathrm{C}$. The pellet was resuspended in
$15 \mathrm{ml}$ resuspension buffer containing $20 \mathrm{mM}$ Tris/ $\mathrm{HCl} \mathrm{pH} \mathrm{8.0,}$ $500 \mathrm{mM} \mathrm{NaCl}, 5 \mathrm{mM}$ imidazole.

Cells were lysed on an ice-water slurry by sonication at $60 \%$ amplitude for $3 \mathrm{~min}$ at $30 \mathrm{~s}$ intervals. The lysate was spun for $15 \mathrm{~min}$ at 19000 r.p.m. and the supernatant loaded onto a $\mathrm{Ni}^{2+}$-Sepharose column (GE Healthcare) preequilibrated with resuspension buffer. The column was washed with resuspension buffer containing $50 \mathrm{mM}$ imidazole and eluted with resuspension buffer containing $400 \mathrm{mM}$ imidazole. The protein was buffer exchanged with a PD-10 column (GE Healthcare) into $50 \mathrm{mM}$ Tris/ $\mathrm{HCl} \mathrm{pH} \mathrm{8.0,} 100 \mathrm{mM} \mathrm{NaCl}$.

\section{Identification of the reaction product}

Purified recombinant $\mathrm{HmbS}$ was heated to $60^{\circ} \mathrm{C}$ for $10 \mathrm{~min}$ on a heat block prior to the assay to deactivate any contaminating UroS. HmbS $(25 \mu \mathrm{g})$ was incubated with $200 \mu \mathrm{M}$ porphobilinogen at $37^{\circ} \mathrm{C}$ in $0.1 \mathrm{M}$ Tris/ $\mathrm{HCl} \mathrm{pH} \mathrm{8.0.} \mathrm{After} 1 \mathrm{~h}$, the reaction was stopped by diluting $10 \times$ into $1 \mathrm{M} \mathrm{HCl}$. The reaction product was oxidized by adding $10 \mu \mathrm{l}$ of a $1 \mathrm{mg} \mathrm{ml}^{-1}$ benzoquinone in methanol and incubating for $60 \mathrm{~min}$. The mixture was run on an HPLC to identify which uroporphyrin isomer was present. Commercial standards of uroporphyrin I and III (Frontier Scientific) were also run to aid identification.

The uroporphyrin I and III isomers were separated on an ACE $5 \mathrm{AQ}$ column, dimensions $250 \times 4.6 \mathrm{~mm}$, using an Agilent 1100

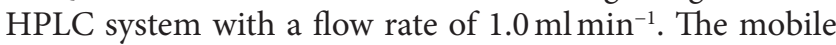
phase was $1 \mathrm{M}$ ammonium acetate $\mathrm{pH} 5.16$ and the organic phase was acetonitrile. A $100 \mu \mathrm{l}$ sample was injected onto the column (temperature $25^{\circ} \mathrm{C}$ ) and the porphyrins were detected by their absorbance at $405 \mathrm{~nm}$. A gradient elution was used rising from 13 to $30 \%$ acetonitrile in $25 \mathrm{~min}$ and held there for a further $5 \mathrm{~min}$. This was adapted from the protocol used elsewhere [18].

\section{Enzyme activity assay}

Enzyme at various concentrations was incubated with $100 \mu \mathrm{M}$ porphobilinogen at $37^{\circ} \mathrm{C}$ in $0.1 \mathrm{M}$ Tris/ $\mathrm{HCl} \mathrm{pH} \mathrm{8.0.} \mathrm{After}$ $25 \mathrm{~min}$, the reaction was stopped by diluting $10 \times$ into $1 \mathrm{M}$ $\mathrm{HCl}$. The reaction product was oxidized by adding $10 \mu \mathrm{l}$ of a $1 \mathrm{mg} \mathrm{ml}^{-1}$ benzoquinone in methanol and incubating for $60 \mathrm{~min}$. Absorbance was read at $405 \mathrm{~nm}$ and the amount of uroporphyrin calculated using the extinction coefficient of $54.8 \times 105 \mathrm{M}^{-1} \mathrm{l}$.

\section{RESULTS}

\section{Complementation studies}

To test if either P. falciparum hemC (encoding HmbS) or P. falciparum 3D7_1247600 (putative coding sequence for UroS) harbour UroS activity, complementation studies were performed to see if either gene could restore growth to a defined hemD $D^{-}$mutant (SASZ31) lacking UroS activity [23]. Two P. falciparum hem $C$ constructs were used, both of which were codon-optimized for expression in E. coli. One contained the full-length hemC gene in a $p E T-3 a$ vector and the other a truncated hem $C$ gene, also in a $p E T-3 a$ vector. The 


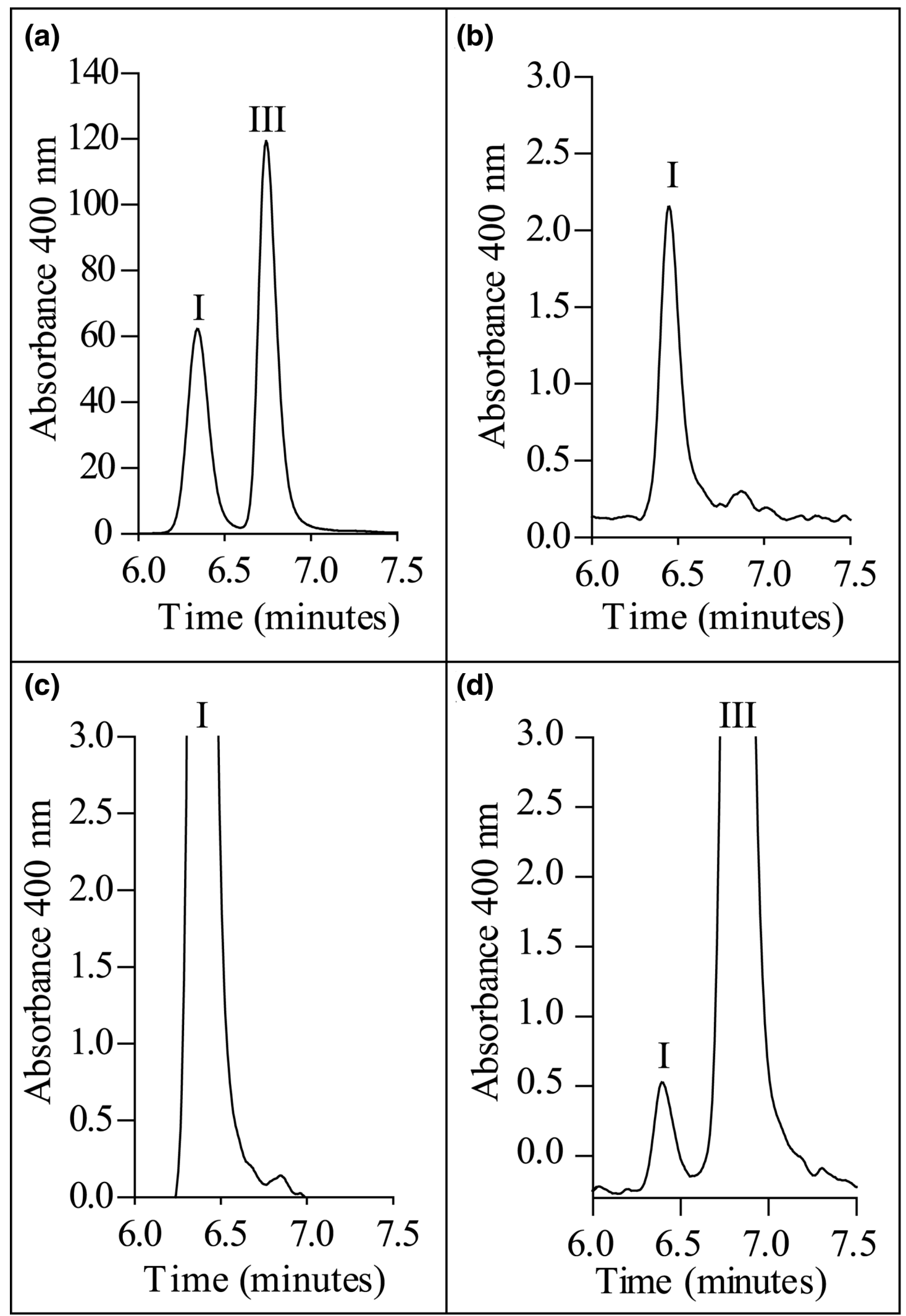

Fig. 2. HPLC analysis of HmbS reaction product. HPLC traces showing (a) commercial standards of uroporphyrin I (left) and III (right) and (b) the oxidized reaction product of $\mathrm{HmbS}$ alone and (c) spiked with uroporphyrin I and (d) uroporphyrin III. 
truncation removed a signal sequence known to hinder gene expression in E. coli and has been shown not to be essential for activity [18].

The hemD $D^{-}$mutant SASZ31 was transformed with these constructs and with control plasmids. The controls included an empty $p E T-3 a$ as a negative control and plasmids harbouring known hemD genes from Bacillus megaterium and E. coli as positive controls. As these control genes were in a $p E T-14 b$ plasmid, an empty $p E T-14 b$ was also used as a further control.

The resulting strains were grown on $\mathrm{LB}$ agar at $37^{\circ} \mathrm{C}$ and the size of colonies was noted at 24 and $48 \mathrm{~h}$. To test for the viability of the colonies after $48 \mathrm{~h}$ they were restreaked onto a fresh $\mathrm{LB}$ agar plate and incubated at $37^{\circ} \mathrm{C}$ for $24 \mathrm{~h}$. The plates were examined for colonies.

The control plasmids harbouring known hemD genes were able to restore normal growth to the hem $D^{-}$mutant. However, the empty vectors and both the $P$. falciparum hem $C$ constructs and the P. falciparum 3D7_1247600 construct were unable to restore normal growth. This demonstrates that neither the $P$. falciparum hem $C$ gene nor $P$. falciparum 3D7_1247600 can complement an E. coli hem $D^{-}$mutant, showing that neither encodes for UroS activity. The results are shown in Table 1.

\section{Protein overproduction in $E$. coli and identification of the reaction product-}

A $p E T-14 b$ construct harbouring the $P$. falciparum hem $C$ gene in frame with an $\mathrm{N}$-terminal hexa-His tag coding sequence was used for protein production in E. coli. The hemC gene was codon-optimized for $E$. coli and lacked the apicoplast localization sequence. The overproduced protein was mostly insoluble but a small quantity of soluble protein was successfully purified to homogeneity from the cell lysate using $\mathrm{Ni}^{2+}$ affinity chromatography. The purity was assessed by SDSPAGE (Fig. S4).

The purified protein was subjected to a $60^{\circ} \mathrm{C}$ heat treatment for $10 \mathrm{~min}$ to deactivate any contaminating UroS. P. falciparum HmbS was demonstrated to be resistant to heat treatment in the original study [18]. The protein was incubated with substrate for $60 \mathrm{~min}$ at $37^{\circ} \mathrm{C}$ and the resulting product was oxidized with $\mathrm{HCl}$ and benzoquinone. This sample was analysed by HPLC to see if the product was uroporphyrin I (corresponding to hydroxymethylbilane) or uroporphyrin III (corresponding to uroporphyrinogen III). Identification was by comparison with commercial standards of uroporphyrin I and III. The reaction product matched the retention time of uroporphyrin I. The results are shown in Fig. 2. As a positive control, analysis of known HmbS and UroS enzymes was also performed (Fig. S5). To confirm that the observed reaction product was enzymatically generated by HmbS, the assay was repeated with different concentrations of enzyme and the reaction product was quantified by absorbance spectrometry. A linear correlation was observed between enzyme concentration and the amount of product formed, which is indicative of enzymatic reactions. The results are presented in Fig. S6.

\section{DISCUSSION}

The claim that $P$. falciparum HmbS has UroS activity [18] has been challenged through complementation studies with a hemD $D^{-}$mutant and HPLC analysis of the reaction product from recombinant enzyme. SASZ31 is a defined hemD ${ }^{-}$ mutant that grows very poorly [23]. Complementation with control hemD genes from B. megaterium and E. coli was able to restore normal growth to the mutant, but $P$. falciparum hem $C$ could not restore normal growth. Because HmbS has an apicoplast localization sequence that hinders expression but is not required for alleged dual activity [18], a truncated gene lacking this sequence was also made. This also failed to complement the mutant.

Furthermore, the truncated HmbS was overproduced in E. coli with an N-terminal hexa-His tag and purified. After incubation with substrate for an hour at $37^{\circ} \mathrm{C}$, the sample was oxidized and run on HPLC along with commercial standards of uroporphyrin I and uroporphyrin III. The HPLC result clearly identified the enzyme's oxidized product as uroporphyrin I. No uroporphyrin III could be detected. These results contradict those previously published [18] where HPLC analysis of the reaction product from native and recombinant HmbS identified the (oxidized) reaction product as uroporphyrin III. This conflict could be explained by the presence of a contaminating UroS in the earlier study. Although the researchers used heat treatment to denature any UroS (HmbS is heat stable but UroS is not), it is possible that any UroS could have refolded and reactivated itself during the $12 \mathrm{~h}$ incubation of heat-treated HmbS with substrate [25-27]. Further, the assay buffer contained additives known to increase the stability of UroS [27].

Our results clearly demonstrate that the previous claim that P. falciparum HmbS contains uroporphyrinogen III synthase (UroS) activity is mistaken [18]. Another report [21] has postulated that UroS activity could reside in the protein encoded by PF3D7_1247600. Our complementation studies have shown that this is also incorrect. It should now be a matter of importance to find the gene that encodes for the real uroporphyrinogen III synthase.

Funding information

This work was supported by Pfizer Global Research and Development.

Acknowledgements

H. Gwawr Davies is thanked for proofreading the manuscript.

Conflicts of interest

The authors declare that there are no conflicts of interest.

References

1. Heinemann IU, Jahn M, Jahn D. The biochemistry of heme biosynthesis. Arch Biochem Biophys 2008:474:238-251.

2. Shemin D, Russell CS. $\delta$-aminolevulinic acid, its role in the biosynthesis of porphyrins and purines 1. J Am Chem Soc 2002;75:4873-4874.

3. Jaffe EK. The remarkable character of porphobilinogen synthase. Acc Chem Res 2016;49:2509-2517.

4. Battersby AR, Fookes CJR, Gustafson-Potter KE, Matcham GWJ, McDonald E. Proof by synthesis that unrearranged 
hydroxymethylbilane is the product from deaminase and the substrate for cosynthetase in the biosynthesis of Uro'gen-III. J Chem Soc Chem Commun;0:1155.

5. Bogorad L. The enzymatic synthesis of porphyrins from porphobilinogen. J Bio Chem 1958;233:501-509.

6. Battersby AR, Fookes CJR, Gustafson-Potter KE, McDonald E, Matcham GWJ. Biosynthesis of porphyries and related macrocycles. Part 18. Proof by spectroscopy and synthesis that unrearranged hydroxymethylbilane is the product from deaminase and the substrate for cosynthetase in the biosynthesis of uroporphyrinogen-III. J Chem Soc Perkin Trans 1;0:2427.

7. Battersby AR, Fookes CJR, Gustafson-Potter KE, McDonald E, Matcham GWJ. Biosynthesis of porphyries and related macrocycles. Part 17. Chemical and enzymic transformation of isomeric aminomethylbilanes into uroporphyrinogens: Proof that unrearranged bilane is the preferred enzymic substrate and detection of a transient intermediate. J Chem Soc Perkin Trans 1;0:2413.

8. Surolia N, Padmanaban G. De novo biosynthesis of heme offers a new chemotherapeutic target in the human malarial parasite. Biochem Biophys Res Commun 1992;187:744-750.

9. Egan TJ. Haemozoin formation. Mol Biochem Parasitol 2008;157:127-136.

10. Ke H, Sigala PA, Miura K, Morrisey JM, Mather MW, et al. The heme biosynthesis pathway is essential for Plasmodium falciparum development in mosquito stage but not in blood stages. J Biol Chem 2014:289:34827-34837

11. Goldberg DE, Sigala PA. Plasmodium heme biosynthesis: To be or not to be essential? PLoS Pathog 2017;13:e1006511.

12. Varadharajan S, Dhanasekaran S, Bonday ZQ, Rangarajan PN, Padmanaban G. Involvement of delta-aminolaevulinate synthase encoded by the parasite gene in de novo haem synthesis by Plasmodium falciparum. Biochem J 2002;367:321-327.

13. Dhanasekaran S, Chandra NR, Chandrasekhar Sagar BK, Rangarajan PN, Padmanaban G. Delta-aminolevulinic acid dehydratase from Plasmodium falciparum: Indigenous versus imported. J Biol Chem 2004;279:6934-6942.

14. Nagaraj VA, Prasad D, Rangarajan PN, Padmanaban G. Mitochondrial localization of functional ferrochelatase from Plasmodium falciparum. Mol Biochem Parasitol 2009;168:109-112.

15. Nagaraj VA, Arumugam R, Prasad D, Rangarajan PN, Padmanaban G. Protoporphyrinogen IX oxidase from Plasmodium falciparum is anaerobic and is localized to the mitochondrion. Mol Biochem Parasitol 2010;174:44-52.
16. Nagaraj VA, Prasad D, Arumugam R, Rangarajan PN, Padmanaban G Characterization of coproporphyrinogen III oxidase in Plasmodium falciparum cytosol. Parasitol Int 2010;59:121-127.

17. Nagaraj VA, Arumugam R, Chandra NR, Prasad D, Rangarajan PN et al. Localisation of Plasmodium falciparum uroporphyrinogen III decarboxylase of the heme-biosynthetic pathway in the apicoplast and characterisation of its catalytic properties. Int J Parasitol 2009:39:559-568.

18. Nagaraj VA, Arumugam R, Gopalakrishnan B, Jyothsna YS Rangarajan PN, et al. Unique properties of Plasmodium falciparum porphobilinogen deaminase. J Biol Chem 2008;283:437-444.

19. Shoolingin-Jordan PM. Porphobilinogen deaminase and uroporphyrinogen III synthase: structure, molecular biology, and mechanism. $J$ Bioenerg Biomembr 1995;27:181-195.

20. Battersby AR, Fookes CJR, McDonald E, Meegan MJ. Biosynthesis of type-III porphyrins: Proof of intact ezymic conversion of the head-to-tail bilane into uro'gen-III by intramolecular rearrangement. J Chem Soc Chem Commun:185.

21. Mohanty S, Srinivasan N. Identification of "missing" metabolic proteins of Plasmodium falciparum: a bioinformatics approach. Protein Pept Lett 2009;16:961-968.

22. Guégan R, Camadro J-M, Saint Girons I, Picardeau M. Leptospira spp. Possess a complete haem biosynthetic pathway and are able to use exogenous haem sources. Mol Microbiol 2003;49:745-754.

23. Chartrand P, Tardif D, Săsărman A. Uroporphyrin- and coproporphyrin I-accumulating mutant of Escherichia coli K12. J Gen Microbiol 1979;110:61-66.

24. Raux E, Leech HK, Beck R, Schubert HL, Santander PJ, et al. Identification and functional analysis of enzymes required for precorrin2 dehydrogenation and metal ion insertion in the biosynthesis of sirohaem and cobalamin in Bacillus megaterium. Biochem $J$ 2003:370:505-516.

25. Jordan PM, Thomas SD, Warren MJ. Purification, crystallization and properties of porphobilinogen deaminase from a recombinant strain of Escherichia coli K12. Biochem J 1988;254:427-435.

26. Alwan AF, Mgbeje BI, Jordan PM. Purification and properties of Uroporphyrinogen III synthase (co-synthase) from an overproducing recombinant strain of Escherichia coli k-12. Biochem $J$ 1989:264:397-402

27. Omata Y, Sakamoto H. Higashimoto Y, Hayashi S, Noguchi M. Purification and characterization of human Uroporphyrinogen III synthase expressed in Escherichia coli. J Biochem 2004;136:211-220.

Edited by: M. Welch and N. Scott

\section{Five reasons to publish your next article with a Microbiology Society journal}

1. The Microbiology Society is a not-for-profit organization.

2. We offer fast and rigorous peer review - average time to first decision is 4-6 weeks.

3. Our journals have a global readership with subscriptions held in research institutions around the world.

4. $80 \%$ of our authors rate our submission process as 'excellent' or 'very good'.

5. Your article will be published on an interactive journal platform with advanced metrics.

Find out more and submit your article at microbiologyresearch.org. 